\title{
Psychosocial adaptation of pregnant women in the conditions of fitness activity
}

\section{Annotation}

In any social environment woman performs one of the most important missions for the human race - the childbearing and reproductive one. This refers the female half of humanity to a certain group of people who understand their significance and necessity. However, not every pregnant woman has personal confidence, while bearing a child. Therefore group fitness classes can contribute to the social identity of a pregnant woman.

Keywords: pregnant women, social identity, fitness
Volume 3 Issue I - 2019

\author{
Aftimichuk Olga \\ State University of Physical Education and Sports, Moldova
}

Correspondence: Aftimichuk Olga, State University of Physical Education and Sports, Moldova, Email aftolig@mail.ru

Received: December 28, 2018 | Published: February 06, 2019

\section{Introduction}

The image of the modern woman over the past decades has undergone dramatic changes not only from the physical, aesthetic point of view, but also from the inside, in a psychosocial sense. Today, women do not want to concede anything to men: it is not surprising to see a female leader in all ranks of positions in various fields of science, culture, education, industry, and politics or not to mention sports field. This position of a woman in society imposes the corresponding requirements on her personal qualities and, which is important for her, her external and internal form. This implies a healthy lifestyle, ${ }^{1-4}$ a high level of efficiency, both mental and physical, strengthening, maintaining and keeping a woman's health, her spiritual and moral condition, and therefore successful psychosocial adaptation to living conditions. However, not only the position in society makes a woman to be active and healthy. We should not forget that, regardless of the functions performed by a woman in the social environment, she performs one of the most important missions for the human race - the childbearing and reproductive one. This refers the female half of humanity to a certain group of people, which, in addition to awareness of the expressed sexual identity, understands its importance and necessity. This process contributes to the formation of social and group identity ${ }^{5-8}$ of a pregnant woman personality (the occurrence of the individual in various communities and awareness of unity with the group environment, in our case with other pregnant women). The future mother lives in her own world, which ends up in the process of carrying and the subsequent birth of the child. All thoughts of a pregnant woman are directed to her baby, to emotions associated with experiencing pregnancy, both positive, as enthusiastic estate, and negative, caused by feelings of anxiety and pressure for herself and the child also. In the given case, an important component of the wellbeing of an expectant mother is health - not only physical, but, not least, mental and psychosocial well-being. ${ }^{5}$

The future health of children and any nation is curled from the state of a woman's health. The activities of women in promoting health and preserving their physical form and image are not only personal, but also social, because society is interested in the birth of healthy children, in a good mental and physical well-being also. However, according to a study by N.A. Anashkina, ${ }^{6}$ the majority of women for various reasons are passive in strengthening their health, although $71 \%$ of them need active relaxation and recreation, as well as, in our opinion, in the development of both the social and group identity of a pregnant woman. The preservation and strengthening of the health of the female population can be promoted by the use of mass forms of recreational physical culture. One of the most attractive motor systems, due to its popularity, accessibility and emotionality, appears to be fitness. The body of a pregnant woman due to the current changes is in a state of constant work. Even in a dormant period, a woman who is preparing to become a mother does a certain amount of work. All body systems of a pregnant woman must adapt to the appropriate level of functioning that is needed to give a new life. Recreational fitness facilities will help a woman cope with the physiological and mental changes that occur during pregnancy. An important component of the motivation of a pregnant woman who has come to the fitness group is represented by the following psychosocial aspects: ${ }^{7}$ communication in a group, the acquisition of new friends in interests, the ability to distract from anxious thoughts and experiences, as well as an increase in self-confidence and strength .d All these processes contribute to the successful psychosocial adaptation of the future mother in the conditions of fitness and the development of both the social and group identity of the pregnant woman in the fitness system.

\section{Materials and methods}

In order to identify the factors contributing to the positive psychosocial adaptation of the personality of women involved in fitness during pregnancy period, we have organized and conducted a sociological study. It has been questioned 46 attendees of fitness clubs in Chisinau, Moldova, where special recreational programs for pregnant women were practiced. The average age of the interviewed women was about 20-30 years. The group of respondents was differentiated according to the social status (Figure 1): workers, students, persons engaged in the household. All the women surveyed are married. An interesting fact is that the majority of working pregnant women combines work with study. This allows us to characterize them as more active, self-reliant - not everyone will be able to combine work with school and having a child. At the same time, in the group of housewives (not all) there is the presence of complexes, low self-esteem, self-doubt, which, of course, affects their physical and social activity. The questionnaire is composed of 64 questions and some of them contain an additional question. Each question comes with various answers, including the own.

All questions of the questionnaire are divided into 3 categories:

I. The first group of questions determines the level of physical fitness of women involved and the effect of fitness on the development of physical qualities.

II. The second group of questions examines the psychological aspect of the influence of fitness programs on the state of the students (issues of a psycho-social nature). 
III. The third group of questions reveals the level of knowledge about fitness of the women that were involved.

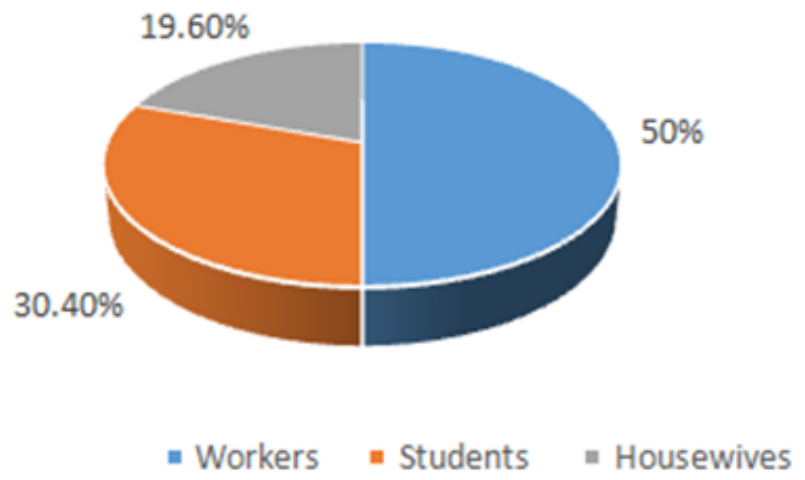

Figure I Respondents composition according to the social status.

\section{Results and discussion}

After we have analyzed the answers of women, we came to the following conclusions. Most women know the indicators that characterize their level of physical condition, $70 \%$ rate their state of health well, but not all (62\%) consulted with their doctor before the start of classes! At the same time, about $70 \%$ were in favor of the necessary presence at the fitness classes for pregnant women of a competent medical professional. All the women surveyed believe that they can control their condition during training, but only half of them will stop practicing if they feel unwell. This indicates a lack of self-control among women involved. $91 \%$ of women were engaged in fitness before pregnancy, confirming the fact that a physically active woman in life prefers to stay this way (of course, not with the same intensity) during pregnancy, and especially after pregnancy. During a fitness workout, they feel a surge of strength, and after exercise - a charge of vivacity and good mood. This group of women seeks to recover faster, to get in shape after childbirth. The first place among physical activity for pregnant women is occupied by: walking, specialized fitness programs and exercises in the water. When asked about the changes associated with fitness activities during pregnancy, $60 \%$ of women answered that they became more confident, $40 \%$ began to lead a healthy lifestyle, $52 \%$ made new friends among other pregnant women in the fitness room. Interest in fitness for pregnant women could increase, first of all, the high quality of classes and the possibility of choosing fitness programs. Many women would make some adjustments to their activities: diversity, breathing exercises, water treatments, ball exercises, and even additional conversations.

$90 \%$ of women practitioners feel quite comfortable in the fitness group and prefer group fitness classes. Most of the respondents feel a psychological, emotional connection with other members of the group, which is explained by the fact of common interests. Half of the women interviewed communicate with other participants in fitness classes outside the hall. $97 \%$ of future mothers trust their trainer and would like to return to him the next pregnancy. All women have noted the changes in their mental state during pregnancy: most of them became more responsible, impressionable, some more irritable and nervous. In this state, fitness training helps to relax and calm down. Among the main motives that prompted women to practice fitness during the pregnancy period, are the following: control of weight gain, increased muscle tone, strengthening and activation of all body systems. Last but not least is the desire to find new friends among the expectant mothers. In general, on the basis of the responses received, it have been identified the factors that form the motivational background for physical exercise and contribute to the positive psychosocial adaptation of the personality of the pregnant woman in the fitness system (Table 1).

Table I Factors contributing to psychosocial adaptation of a pregnant woman personality under fitness conditions

\begin{tabular}{|c|c|c|}
\hline No. & Factors & Content \\
\hline I & Cosmetic Aspect & $\begin{array}{l}\text { Weight control during and after pregnancy, the formation of muscle corset, maintaining muscle tone, } \\
\text { improving posture, gait, etc. }\end{array}$ \\
\hline 2 & Medical aspects and hygienic skills & Improving immunity, reducing the frequency of diseases, avoiding bad habits, proper diet, sleep, etc. \\
\hline 3 & Physical performance & $\begin{array}{l}\text { The need for physical activity, physical education, fitness, the achievement and maintenance of certain } \\
\text { results, a certain level of physical condition, the development of physical qualities, etc. }\end{array}$ \\
\hline 4 & Neuropsychiatric stability and well-being & Enjoying classes, good mood, great well-being, resistance to stress, etc. \\
\hline 5 & Psychosocial aspects & $\begin{array}{l}\text { Self-confidence and confidence in their abilities, increasing self-esteem, getting rid of complexes related } \\
\text { to appearance, communication with the participants of the fitness group and, as a result, new friends, } \\
\text { social circle, etc. }\end{array}$ \\
\hline
\end{tabular}

\section{Conclusion}

This way, successful psychosocial adaptation of the personality of a pregnant woman in a fitness system allows you to realize the need and benefit of health-improving physical culture, feeling healthy, fully-developed, mentally stable personality and make the right choice in favor of an active lifestyle that will help during pregnancy and after giving birth.

\section{Acknowledgments}

None.

\section{Conflicts of interest}

The auhtor declares that there is no conflicts of interest.

\section{References}

1. Ornish D. The Spectrum: A Scientifically Proven Program to Feel Better, Live Longer, Lose Weight, Gain Health. New York: Ballantine. 2008.

2. Paffenbarger RS, Olsen E. Lifefit: An Effective exercise program for optimal health and a longer life. Champaign, IL: Human Kinectics. 1996.

3. Reiss S. Multifaceted nature of intrinsic motivation: The theory of 16 basic desires. Review of General Psychology. 2004;8(3):179-193. 
4. Robbins J. Healthy at 100: The Scientifically Proven Secrets of the World's Healthiest and Longest-Lived Peoples. New York: Ballantine Books. 2007. 357 p

5. Aftimichuk OE. Peculiarities of the organization and conduct of trainings on aerobics with different contingent of people. Pregnant women. In: Aerobics for health. Theory and methodology. Chisinau: „Valinex” SRL: 2011. p. 220-229

6. Anashkina NA. Recreational and recreational activities of women in modern socio-cultural conditions. In: Jubilee collection of scientific papers of young scientists of RSAPE. Moscow: 2004. p. 5-9.
7. Berezovska-Florintsy T, Aftimichuk OE. Social identity of the personality of a modern person in the conditions of fitness. In: Probleme actuale ale teoriei şi practicii culturii fizice: Conf. ştiinţ. internaţ. stud. Ed. A XII-a. Chişinău: 2008. p. 150-157.

8. Kamenskaya EN. Social psychology: Lecture notes edition 2. Rostov n/D: Phoenix. 2006. 